IAU Symposium

20 - 24 August 2012

Beijing, China
Proceedings of the International Astronomical Union

\section{Astrophysics from Antarctica}

Edited by

\section{Michael G. Burton}

Xiangqun Cui

Nicholas F.H.Tothill

ISSN 1743-9213

International Astronomical Union

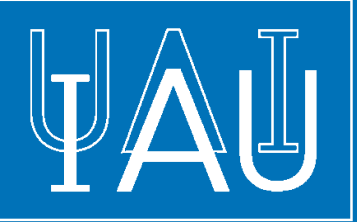

CAMBRIDGE UNIVERSITY PRESS

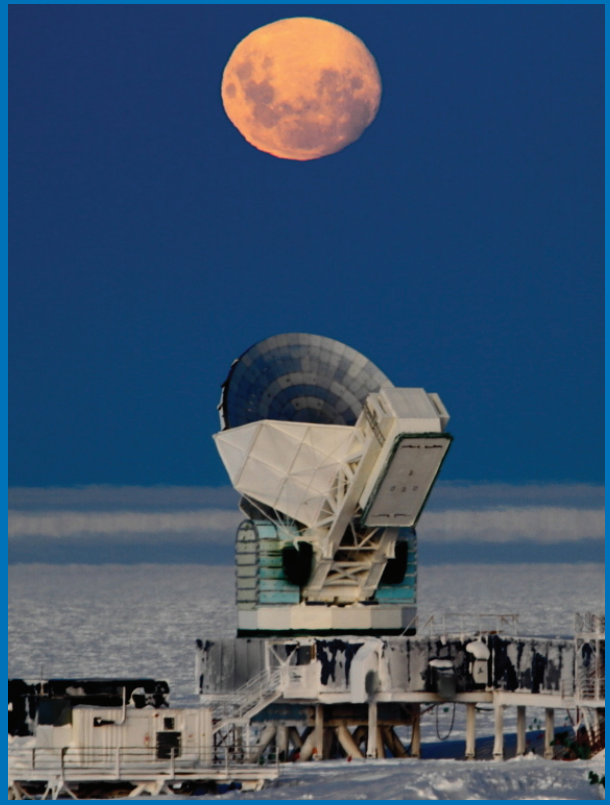

Published online by Cambridge University Press 


\section{ASTROPHYSICS FROM ANTARCTICA}

IAU SYMPOSIUM No. 288

\section{COVER ILLUSTRATION: THE SOUTH POLE TELESCOPE}

The $10 \mathrm{~m}$ diameter South Pole Telecope (or SPT), seen under the light of the full Moon on a clear night, just before sunrise at the Pole. The SPT is located in the Dark Sector Laboratory (DSL) at the Amundsen-Scott South Station, $1 \mathrm{~km}$ away from the geographic South Pole.

The SPT is being used to observe the Cosmic Microwave Background Radiation, surveying a 2,500 square degree area of sky. The science objectives include searches for galaxy clusters using the Sunyaev Zel'dovic effect and making the highest angular resolution measurements of the CMB power spectrum. The results are being used to constrain models for Dark Energy and other cosmological parameters.

Photograph taken by Daniel Luong-Van in September 2010, wintering scientist for the SPT during 2010 and 2011. The picture was taken with a Canon 7D camera about $1.5 \mathrm{~km}$ away from the telescope in order to make the Moon and the SPT comparable in scale. It is interesting to note that the band of white is not cloud but is a mirage, being the reflection off the snow surface. 
IAU SYMPOSIUM PROCEEDINGS SERIES

\author{
Chief Editor
}

THIERRY MONTMERLE, IAU General Secretary

Institut d'Astrophysique de Paris, 98bis, Bd Arago, 75014 Paris, France

montmerle@iap.fr

\title{
Editor
}

PIERO BENVENUTI, IAU Assistant General Secretary University of Padua, Dept of Physics and Astronomy, Vicolo dell'Osservatorio, 3, 35122 Padova, Italy piero.benvenuti@unipd.it 
INTERNATIONAL ASTRONOMICAL UNION

UNION ASTRONOMIQUE INTERNATIONALE

International Astronomical Union

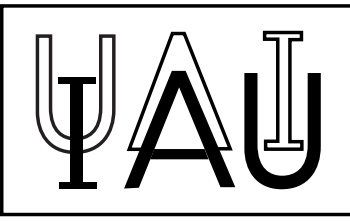

\section{ASTROPHYSICS FROM ANTARCTICA}

\section{PROCEEDINGS OF THE 288th SYMPOSIUM OF THE INTERNATIONAL ASTRONOMICAL UNION HELD IN BEIJING, CHINA} AUGUST 20-24, 2012

Edited by

MICHAEL G. BURTON

University of New South Wales, AUSTRALIA

XIANGQUN CUI

Nanjing Institute for Astronomical Optics and Technology, CHINA

and

NICHOLAS F. H. TOTHILL

University of Western Sydney, AUSTRALIA 
CAMBRIDGE UNIVERSITY PRESS

The Edinburgh Building, Cambridge CB2 2RU, United Kingdom

32 Avenue of the Americas, New York, NY 10013 2473, USA

10 Stamford Road, Oakleigh, Melbourne 3166, Australia

(C) International Astronomical Union 2013

This book is in copyright. Subject to statutory exception and to the provisions of relevant collective licensing agreements, no reproduction of any part may take place without the written permission of the International Astronomical Union.

First published 2013

Printed in the UK by MPG Books Ltd

Typeset in System $\mathrm{LT}_{\mathrm{EX}} 2_{\varepsilon}$

A catalogue record for this book is available from the British Library

Library of Congress Cataloguing in Publication data

This journal issue has been printed on FSC-certified paper and cover board. FSC is an independent, non-governmental, not-for-profit organization established to promote the responsible management of the worlds forests. Please see www.fsc.org for information.

ISBN 9781107033771 hardback

ISSN $1743-9213$ 


\section{Table of Contents}

Preface ............................. ix M. Burton and X. Cui

Organisation and Acknowledgements .................... xii

Conference Photograph $\ldots \ldots \ldots \ldots \ldots \ldots \ldots \ldots \ldots \ldots \ldots \ldots \ldots \ldots \ldots \ldots \ldots \ldots \ldots$ xiii

List of Participants $\ldots \ldots \ldots \ldots \ldots \ldots \ldots \ldots \ldots \ldots \ldots \ldots \ldots \ldots \ldots \ldots \ldots \ldots \ldots$

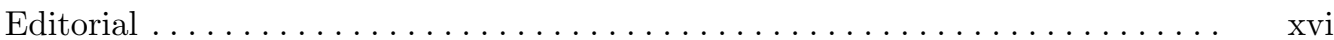

M. G. Burton, X. Cui, \& N. F. H. Tothill

Review of Antarctic astronomy....................... 1

J. W. V. Storey

Autonomous observatories for the Antarctic plateau . . . . . . . . . .

J. S. Lawrence, M. C. B. Ashley, \& J. W. V. Storey

Site characteristics of the high Antarctic plateau $\ldots \ldots \ldots \ldots \ldots \ldots \ldots$ M. C. B. Ashley

Dome Fuji Seeing - Summer Results and Future Winter-over Observations ..... H. Okita, N. Takato, T. Ichikawa, C. S. Bonner, M. C. B. Ashley, J. W. V. Storey and the $51^{\text {st }}$ and $52^{\text {nd }}$ JARE Dome Fuji teams

A worldwide comparison of the best sites for submillimetre astronomy . . . . . . P. Tremblin, N. Schneider, V. Minier, G. Al. Durand \& J. Urban

Winter sky brightness and cloud cover at Dome A, Antarctica ...........

A. M. Moore, Y. Yang, J. Fu, M. C. B. Ashley, X. Cui, L. Feng, X. Gong,

Z. Hu, J. S. Lawrence, D. M. Luong-Van, R. Riddle, Z. Shang, G. Sims,

J. W. V. Storey, N. F. H. Tothill, T. Travouillon, L. Wang, H. Yang,

J. Yang, X. Zhou \& Z. Zhu

First look at HRCAM images from Dome A, Antarctica . . . . . . . . . .

G. Sims, M. C. B. Ashley, X. Cui, L. Feng, X. Gong, Z. Hu, J. S. Lawrence,

D. M. Luong-Van, Z. Shang, J. W. V. Storey, N. Tothill, L. Wang, H. Yang,

J. Yang, X. Zhou \& Z. Zhu

CMB anisotropy science: a review $\ldots \ldots \ldots \ldots \ldots \ldots \ldots \ldots \ldots \ldots$

A. Challinor

Precision CMB Measurements from Long Duration Stratospheric Balloons: Towards B-modes and Inflation. . . . . . . . . . . . . . . . W. C. Jones

A CMB B-mode Search with Three Years of BICEP Observations . . . . . . . . . C. Bischoff for the BICEP Collaboration

CMB Polarization with BICEP2 and Keck-Array ...............

C. Pryke for the BICEP2 and Keck-Array Collaborations 
Toward a 10,000-element B-Mode Experiment . . . . . . . . . . . . . .

C.-L. Kuo for the BICEP3 and POLAR1 Collaborations

Neutrino Astronomy: An Update . . . . . . . . . . . . . . . . .

F. Halzen

The Path from AMANDA to IceCube

A. Karle

The IceCube Neutrino Telescope $\ldots \ldots \ldots \ldots \ldots \ldots \ldots \ldots \ldots \ldots$

T. K. Gaisser for the IceCube Collaboration

The Askaryan Radio Array . . . . . . . . . . . . . . . . . . .

K. D. Hoffman

Cometary dust in Antarctic micrometeorites.

N. Imae

Antarctic meteorites and the origin of planetesimals and protoplanets.......

A. Yamaguchi

$\mathrm{THz}$ Observations of the Cool Neutral Medium ................

J. M. Dickey

The Exploration of the ISM from Antarctica $\ldots \ldots \ldots \ldots \ldots \ldots \ldots$ M. G. Wolfire

Submillimeter Astronomy from the South Pole (AST/RO)

A. A. Stark

The Balloon-borne Large Aperture Submillimetre Telescope (BLAST) and

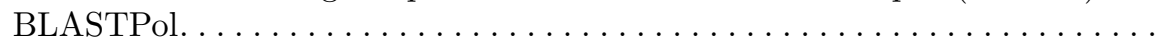

E. Pascale for the BLAST and BLASTPOL collaboration

Dome Fuji Station in East Antarctica and the Japanese Antarctic Research

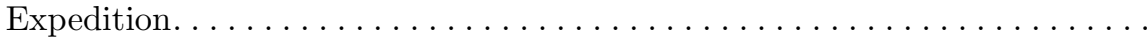

K. Shiraishi

The US Long Duration Balloon Facility at McMurdo Station . . . . . . . . . . . $W$. V. Jones

The French-Italian Concordia Station $\ldots \ldots \ldots \ldots \ldots \ldots \ldots \ldots \ldots$ D. Mekarnia \& Y Frenot

Winterover scientists in Antarctic Astrophysics N. F. H. Tothill \& C. L. Martin

Astronomy from 80 Degrees North on Ellesmere Island, Canada . . . . . . . . . . 194 E. Steinbring

Sub-mm VLBI from the Arctic — Imaging Black Holes. $M$. Inoue and the Greenland Telescope team

$225 \mathrm{GHz}$ Atmospheric Opacity Measurements from Two Arctic Sites. . . . . . . . . S. Matsushita, M.-T. Chen, P. Martin-Cocher, K. Asada, C.-P. Chen, M. Inoue, S. Paine, D. Turner \& E. Steinbring

Precision CMB measurements with long-duration stratospheric balloons: activities

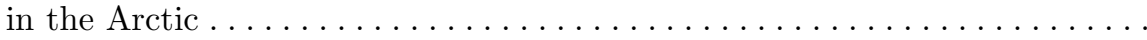
P. de Bernardis, S. Masi for the OLIMPO and LSPE teams 
Present and Future Observations of Earthshine from Antarctica. . . . . . . . . .

D. Briot, L. Arnold \&S S. Jacquemoud

Time domain astronomy from Dome C: results from ASTEP . . . . . . . . .

J.-P. Rivet, L. Abe, K. Agabi, M. Barbieri, N. Crouzet, I. Goncalves,

T. Guillot, D. Mekarnia, J. Szulagyi, J.-B. Daban, C. Gouvret,

Y. Fantei-Caujolle, F.-X. Schmider, T. Furth, A. Erikson, H. Rauer,

F. Fressin, A. Alapini, F. Pont 8 S. Aigrain

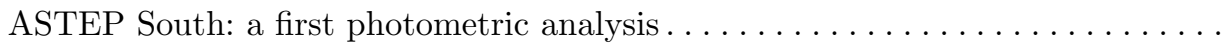

N. Crouzet, T. Guillot, D. Mékarnia, J. Szulágyi, L. Abe, A. Agabi,

Y. Fantë̈-Caujolle, I. Gonçalves, M. Barbieri, F.-X. Schmider, J.-P. Rivet,

E. Bondoux, Z. Challita, C. Pouzenc, F. Fressin, F. Valbousquet, A. Blazit,

S. Bonhomme, J.-B. Daban, C. Gouvret, D. Bayliss, G. Zhou and the ASTEP team

Progress and Results from the Chinese Small Telescope ARray (CSTAR) . .....

X. Zhou, M. C. B. Ashley, X. Cui, L. Feng, X. Gong, J. Hu, Z. Jiang,

C. A. Kulesa, J. S. Lawrence, G. Liu, D. M. Luong-Van, J. Ma, L. M.

Macri, Z. Meng, A. M. Moore, W. Qin, Z. Shang, J. W. V. Storey, B. Sun,

T. Travouillon, C. K. Walker, J. Wang, L. Wang, L. Wang, S. Wang,

J. Wu, Z. Wu, L. Xia, J. Yan, J. Yang, H. Yang, Y. Yao, X. Yuan, D. York,

H. Zhang, Z. Zhang, J. Zhou, Z. Zhu \& H. Zou

Next Generation Deep $2 \mu \mathrm{m}$ Survey ....................

J. Mould

A European vision for a "Polar Large Telescope" project . . . . . . . . . . . .

L. Abe, N. Epchtein, W. Ansorge, S. Argentini, I. Bryson, M. Carbillet, G.

Dalton, C. David, I. Esau, C. Genthon, M. Langlois, T. Le Bertre, R.

Lemrani, B. Le Roux, G. Marchiori, D. Mékarnia, J. Montnacher, G.

Moretto, P. Prugniel, J.-P. Rivet, E. Ruch, C. Tao, A. Tilquin \& I. Vauglin

Dome Fuji in Antarctica as a Site for Infrared and Terahertz Astronomy...... .

M. Seta, N. Nakai, S. Ishii, M. Nagai, Y. Miyamoto, T. Ichikawa, N. Takato \&) H. Motoyama

Opportunities for Terahertz Facilities on the High Plateau . . . . . . . . . .

C. A. Kulesa, M. C. B. Ashley, Y. Augarten, C. S. Bonner, M. G. Burton,

L. Bycroft, J. Lawrence, D. H. Lesser, J. Loomis, D. M. Luong-Van, C. L.

Martin, C. McLaren, S. Stapleton, J. W. V. Storey, B. J. Swift, N. F. H.

Tothill, C. K. Walker \& A. G. Young

Optical Interferometry from the Antarctic.

P. Tuthill

Preliminary design of the Kunlun Dark Universe Survey Telescope (KDUST) . .

X. Yuan, X. Cui, D.-Q. Su, Y. Zhu, L. Wang, B. Gu, X. Gong \& X. Li

The SCAR Astronomy \& Astrophysics from Antarctica Scientific Research

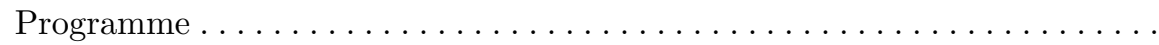

J. W. V. Storey, L. Abe, M. Andersen, P. Anderson, M. Burton, X. Cui,

T. Ichikawa, A. Karle, J. Lloyd, S. Masi, E. Steinbring, T. Travouillon,

P. Tuthill \& H. Zhou

Preliminary daytime seeing monitoring at Dome A, Antarctica .......... C. Pei, Z. Li, H. Chen $\&$ X. Yuan 
Testing the bimodal distribution of long gamma-ray bursts in the cosmological

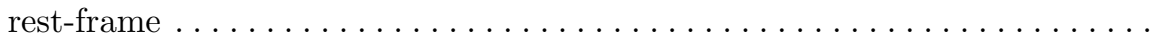

C. Vásconez, N. Vásquez \& E. López

Dome $\mathrm{C}$ site testing: long term statistics of integrated optical turbulence parame-

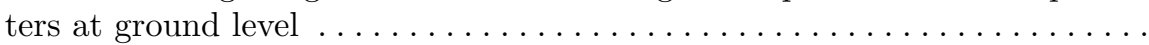
E. Aristidi, A. Agabi, E. Fossat, A. Ziad, L. Abe, E. Bondoux, G. Bouchez, Z. Challita, F. Jeanneaux, D. Mékarnia, D. Petermann \& C. Pouzenc

Airglow and Aurorae from Dome A, Antarctica ................. G. Sims, M. C. B. Ashley, X. Cui, J. R. Everett, L. Feng, X. Gong, S. Hengst, Z. Hu, J. S. Lawrence, D. M. Luong-Van, A. M. Moore, R. Riddle, Z. Shang, J. W. V. Storey, N. Tothill, T. Travouillon, L. Wang, H. Yang, J. Yang, X. Zhou \& Z. Zhu

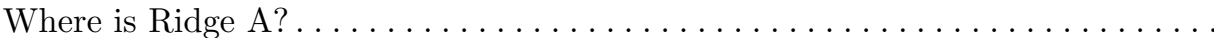

G. Sims, C. Kulesa, M. C. B. Ashley, J. S. Lawrence, W. Saunders \& J.W. V. Storey

Shape measurement by using basis functions. 306

G. Li, B. Xin \& W. Cui

A Multi-Aperture Scintillation Sensor for Dome A, Antarctica............ H. Chen, C. Pei, \& X. Yuan

304

Design and field testing of the Fish-Eye lens for optical atmospheric observations I. Syniavskyi, Y. Ivanov, S. Chernous \& F. Sigernes

Solar Eclipses Observed from Antarctica . . . . . . . . . . . . . . . . . . J. M. Pasachoff

SCIDAR: an optical turbulence profiler for Dome A............... L.-Y. Liu, Y.-Q. Yao, J. Vernin, M. Chadid, H.-S. Wang \& Y.-P. Wang

SONG China project - participating in the global network .......... L. Deng, Y. Xin, X. Zhang, Y. Li, X. Jiang, G. Wang, K. Wang, J. Zhou, Z. Yan \& Z. Luo

Photometry of Variables from Dome A, Antarctica ................. L. Wang, L. M. Macri, L. Wang, M. C. B. Ashley, X. Cui, L. L. Feng, X. Gong, J. S. Lawrence, Q. Liu, D. Luong-Van, C. R. Pennypacker, Z. Shang, J. W. V. Storey, H. Yang, J. Yang, X. Yuan, D. G. York, X. Zhou, Z. Zhu \& Z. Zhu

Secular variation and fluctuation of GPS Total Electron Content over Antarctica R. Jin E S. Jin

Electron-antineutrino disappearance seen by Daya Bay reactor neutrino experiment $R$. Wang, on behalf of the Daya Bay Collaboration

Test and Commissioning of the AST3-1 Control System . . . . . . . . . . X. Li \& D. Wang

Atmospheric calibration for submillimeter and terahertz observations ...

X. Guan, J. Stutzki \& Y. Okada

Classification of Quasars and Stars by Supervised and Unsupervised Methods . . Y. Zhang, Y. Zhao, H. Zheng \& X.-B. Wu 


\section{Preface}

\section{Overview}

The remarkable environment of Antarctica offers many advantages for astronomical observations. Over the past two decades this field of scientific endeavour has developed dramatically and Antarctic-based observatories now regularly contribute to front line astrophysical research, in particular in the measurement of the cosmic microwave background radiation. Scientific stations where astronomy is a major focus now exist at five locations on the Antarctic plateau, as well as on the Antarctic coast, and are operated as international facilities. This Symposium examined the contributions to astrophysics that Antarctic telescopes have made. It reviewed our understanding of the Antarctic environment, in particular that on the high Antarctic plateau. It considered the developments taking place across the continent. It also looked at the parallel opportunities offered by the Arctic environment. Finally, the Symposium examined the science that may be best addressed by future facilities on the Antarctic continent.

\section{The Science}

Over the past decade astronomy has matured as one of the fields of science that is being pursued in Antarctica, notably through high angular resolution studies of the cosmic microwave background, including the demonstration that the Universe is flat (de Bernardis et al. 2000), the first measurements of polarization of the CMB E-mode (Kovac et al. 2002), measurement of the kinematic and thermal SZ-effect in galaxy clusters (Staniszewski et al. 2009) and the demonstration that at high- $l$ the background radiation is dominated by emission from dusty galaxies (Leuker et al. 2010). Several experiments are now being conducted, worldwide, to search for the B-modes of the CMB, with a large part of this effort taking place in Antarctica, using both ground-based and balloon-borne experiments. Over the same time period there have been science programs conducted in Antarctica from the optical to the sub-millimetre wavebands, and also a range of facilities constructed for high energy astrophysics, particularly for neutrino detection. These science achievements of astronomy in Antarctica have been summarised in the recent review of the field by Burton (2010).

\section{The Stations}

Concurrently, major infrastructure has been developed over the Antarctic plateau, undertaken by several different nations. The US have completed a major upgrade to the Amundsen-Scott South Pole station, as well as the construction of the first neutrino telescope (IceCube), in addition to an on-going CMB program. France and Italy have completed the building of the Concordia Station at Dome C, and have wintered personnel there since 2005. Dome A was first visited by humans that year and China has begun the construction of Kunlun station at the site. Dome Fuji station at Dome F has had personnel winter over for ice-core drilling, and astronomical site testing has recently been initiated there. Ridge A was first visited this very year, and a fully robotic telescope installed by the USA and Australia. Long durations balloons equipped with astronomical payloads are now being regularly launched from the US McMurdo station on the Antarctic coast with further extended missions under development. 


\section{The Environment}

Our understanding of the Antarctic environment as it pertains to the conduct of astronomical observations has improved dramatically over the past decade. Through the use of autonomous observatories at the South Pole, Dome C and Dome A, considerable quantitative information is now available on the properties and behaviour of the sky background and atmospheric transparency, as well as its stability over the high plateau. In particular, the special properties of the narrow surface boundary layer are being revealed. The median thickness of the boundary layer is now known to be less than $15 \mathrm{~m}$ thick in winter at the highest places on the plateau, and is often only half that height (e.g. Bonner et al. 2010). Further development of automated observatories at Dome A, Dome $\mathrm{F}$ and Ridge $\mathrm{A}$ is underway to expand this knowledge of the Antarctic environment. Test-bed astronomical facilities are also being operated at Domes A and C, prior to the completion of full infrastructure at these sites.

\section{The Astronomy}

Astrophysics has now been conducted in Antarctica in the optical, infrared, terahertz (or far-infrared) and sub-millimetre portions of the spectrum with a variety of small-scale facilities, undertaking studies mostly of stars, gas and dust in the Galaxy. Telescopes such as the $60 \mathrm{~cm}$ infrared SPIREX and the $1.7 \mathrm{~m}$ sub-millimetre AST/RO have demonstrated the ability to undertake new science in these bands in Antarctica. Of particular note is the recent demonstration of the opportunity for time series investigations in the optical domain with an extremely high cadence and duty cycle (the CSTAR experiment; Yang et al. 2010) at Dome A, and at Dome C for precision photometry to be undertaken (sIRAIT; Strassmeier et al. 2008) and the monitoring of exoplanet transits (ASTEP-South; Crouzet et al. 2010). Thermal infrared large scale repeated surveys, such as proposed for the PLT (Dome C) and KDUST (Dome A) telescopes, would also greatly benefit from Antarctic atmospheric conditions.

\section{The Role of the IAU}

The IAU has a special role in furthering the development of astronomy in Antarctica because of the unique political situation on the continent. No country owns Antarctica. International collaboration and co-ordination is the modus operandi for Antarctic science. This is facilitated through SCAR, a fellow ICSU body with the IAU with specific interests for Antarctic science. IAU recently affiliated with SCAR, the ninth scientific union to do so. Astronomy is not, however, a "traditional" Antarctic science. It faces particular difficulty in attracting funding for infrastructure as a result of many national funding arrangements. The IAU can play an important role in furthering astronomy in Antarctica through supporting the holding of a Symposium in the field, so giving recognition to both the maturing of the field as well as to the science potential it offers.

\section{Chinese Plans for Antarctica}

China, the host country for the IAU's XXVIII GA in 2012, also has a special interest in the development of astronomy in Antarctica at its newly established Kunlun station, following the first human visit to the site by China in just 2005. The first astronomical experiments are now taking place there during the construction phase of the station. Under the Chinese government's $12^{\text {th }} 5$-year plan two major facilities would operate at 
Dome A when the station is completed: for the optical / infrared the $2.5 \mathrm{~m}$ KDUST telescope and for the $\mathrm{THz}$ waveband the $5 \mathrm{~m}$ DATE5 telescope.

\section{Opportunities in the Arctic}

The Arctic regions also offer several sites with comparable characteristics to Antarctica, notably in Greenland and in Ellesmere Island in Canada. These are high $(>2,500 \mathrm{~m})$, dry locations on the summits of large islands located in the Arctic Ocean. Several sites are now under investigation there regarding their suitability for astronomical observations. Sub-orbital programs are also being planned for the Arctic regions, so as to achieve the full-sky coverage needed for large-scale polarization surveys of the CMBR. The science opportunities presented by Arctic observatories was also a theme at the meeting.

\section{Looking to the Future}

"Astrophysics from Antarctica" examined all the above themes. The Symposium reviewed our current understanding of the Antarctic environment as it pertains to astronomical observations. It looked at the major achievements of astrophysics in Antarctica to date, in particular with the CMB, but also in high energy astrophysics (neutrinos), radio astronomy (sub-millimetre and $\mathrm{THz}$ ) and optical/IR astronomy. The Symposium also examined international developments in Antarctica, in particular at the high plateau stations, and the science themes that are emerging for them. In turn, these have many common needs for infrastructure, as well as in challenges that need to be met. To facilitate their furtherance, the Symposium also overviewed this international endeavour, including both the science programs being conducted through national Antarctic programs and the facilities being built or considered for their pursuit. Large international projects are increasingly driving developments in astronomy, and Antarctica and its method of government provides a locale where a "World Observatory" might be built. A particular focus was given to where Antarctic facilities can best contribute to addressing the big science questions posed for astronomy in many national reviews. The Symposium ended by seeking a vision for the future development of international facilities on the continent.

This Volume presents the papers delivered on all these subject matters at IAU Symposium 288, "Astrophysics from Antarctica", held in the China National Convention Center in Beijing from 20-24 August, 2012.

Michael Burton and Xiangqun Cui, Co-chairs SOC, IAU Symposium 288

Sydney \& Nanjing, November 1, 2012

\section{References}

Bonner, C. S., et al. 2010, PASP, 122, 1122

Burton, M. G. 2010, A\& A Rev., 18, 417

Crouzet, N., et al. 2010, A\&A A, 511, A36

de Bernardis, P., et al. 2000, Nature, 404, 955

Kovac, J. M. et al. 2002, Nature, 420, 772

Lueker, M., et al. 2010, ApJ, 719, 1045

Staniszewski, Z., et al. 2009, ApJ, 701, 32

Strassmeier, K. G., et al. 2008, A\&SA, 490, 287

Yang, H., et al. 2010, PASP, 122, 490 


\section{ORGANISATION AND ACKNOWLEDGEMENTS}

\section{Scientific Organising Committee}

Michael Burton (Australia) (co-chair)

Xiangqun Cui (China) (co-chair)

Leo Bronfman (Chile)

Nicolas Epchtein (France)

Peter Gorham (USA)

Takashi Ichikawa (Japan)

Doug Johnstone (Canada)

John Kovac (USA)

Silvia Masi (Italy)

Young Minh (Korea)

Klaus Strassmeier (Germany)

Ji Yang (China)

Zhaohui Shang (China)

\section{Editors of the Proceedings}

Michael Burton (Australia)

Xiangqun Cui (China)

Nicholas Tothill (Australia)

\section{Acknowledgements}

The Symposium was sponsored and supported by the IAU's Divisions IX (Optical and Infrared Techniques), X (Radio Astronomy) and XI (Space and High-Energy Astrophysics), as well as by the IAU Working Group for the Development of Antarctic Astronomy. In addition, the IAU's cognate ICSU body SCAR (Scientific Committee for Antarctic Research), the Chinese Astronomical Society and the National Research Council of Canada's Herzberg Institute of Astrophysics, gave their support for the Symposium.

The Symposium Dinner was sponsored by the Nanjing Institute of Astronomical Optics and Technology together with Purple Mountain Observatory in China.

The Symposium website, at www.phys.unsw.edu.au/IAUS288, was supported by the School of Physics at the University of New South Wales in Australia. 
CONFERENCE PHOTOGRAPH

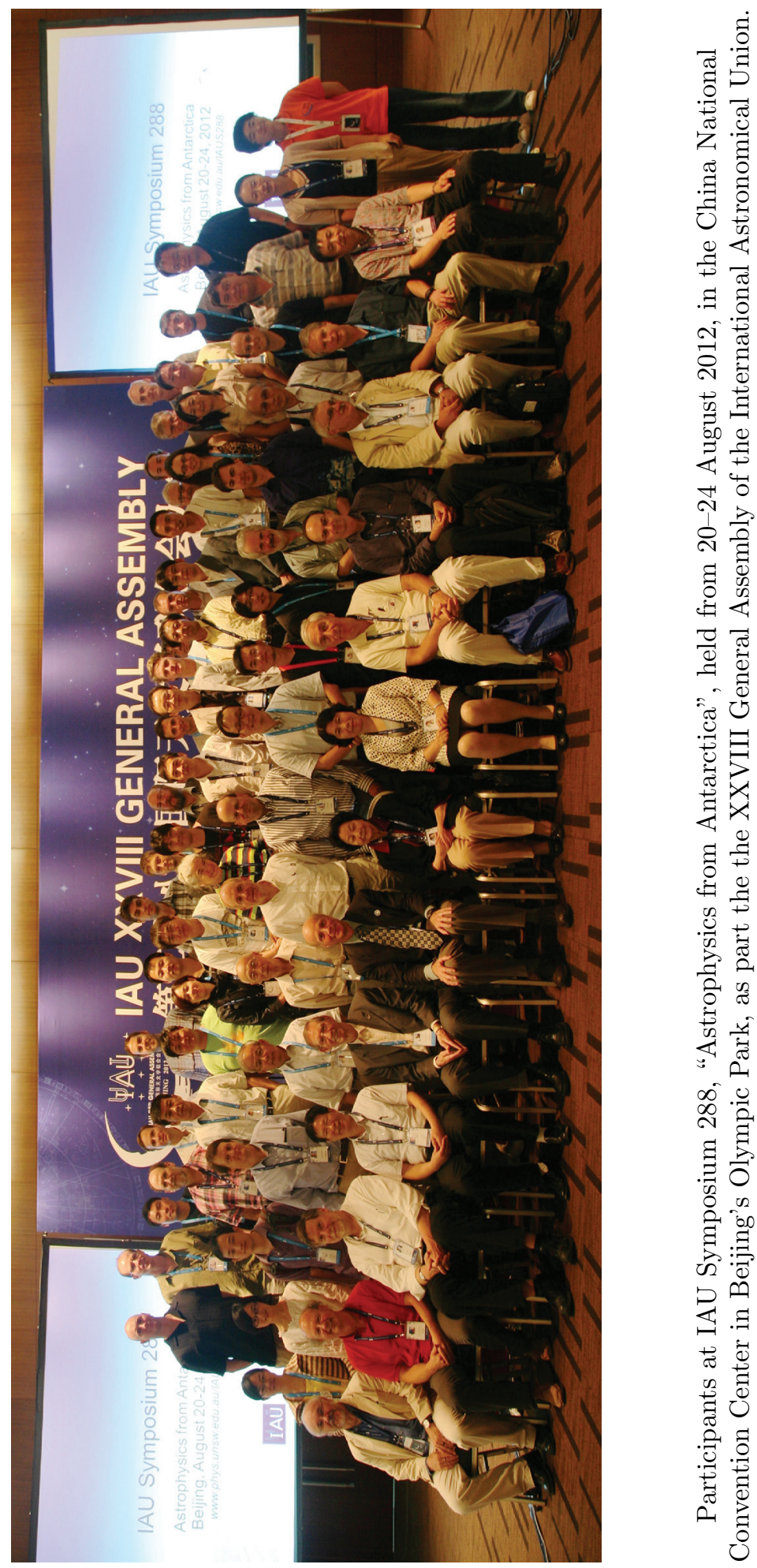




\section{Participants}

Yl Ai

Karle Albrecht

Tilquin Andre

Moore Anna

Challinor Anthony

Stark Antony

Eric Aristidi

Steve Barwick

Sabogal Beatriz

Kumthekar Bhagvat

Thide Bo

Benson Bradford

Danielle Briot

ten Brummelaar Theo

Kuo Chao-Lin

Tao Charling

Huang Chen

Liu Chengzhi

Vasconez Christian

Martin Christopher

Pun Chun Shing Jason

Sarah Church

Pryke Clem

Bischoff Colin

Kulesa Craig

Licai Deng

Mekarnia Djamel

Bock Douglas

Steve Durst

Pascale Enzo

Steinbring Eric

Fossat Eric

Wang Gang

Zhao Gang

Sims Geoff

Lombardi Gianluca

Novak Giles

Durand Gilles

Zhao Gong-Bo

Murray Graham

Liu Guoqing

Francis Halzen

Zinnecker Hans

Zuo Heng

Okita Hirofumi

Matsuo Hiroshi

Wang Hongchi

Wang Hongchi
Sylwester Janusz

Sylwester Janusz
Hamilton Jean-Christophe

Rivet Jean-Pierre

Yang Ji

Wu Jianghua

Fu Jianning

Rui Jin

Nam Jiwoo

Greiner Jochen

Dickey John

Storey John

Lawrence Jon

Vernon Jones

Navarrete Julio

Hoffman Kara

Strassmeier Klaus

John Kovac

Guoliang Li

Xiaoyan Li

Yuansheng Li

Wang Lifan

Liu Liyong

Damé Luc

A be Lyu

Inoue Makoto

Ruiqing Mao

Ruiqing Mao
Sarazin Marc

Sergio Marenss

Wolfire Mark

McCaughrean Mark

McAuley Mark

Reina Maruyama

Seta Masumichi

Colless Matthew

Ashley Michael

Burton Michael

Young Minh

Jeremy Mould

Imae Naoya

Tothill Nicholas

Crouzet Nicolas

Sharp Nigel ayl@ynao.ac.cn

karle@icecube.wisc.edu

tilquin@cppm.in2p3.fr

amoore@astro.caltech.edu a.d.challinor@ast.cam.ac.uk aas@cfa.harvard.edu aristidi@unice.fr

barwick@HEP.ps.uci.ed bsabogal@uniandes.edu.co

bkumthekar@gmail.com bt@irfu.se

bbenson@kicp.uchicago.ed

danielle.briot@obspm.

theo@chara-array.org

clkuo@stanford.edu

tao@cppm.in2p3.fr hc@shao.ac.cn lcz@cho.ac.cn

cvasconez@gmail.com

Chris.Martin@oberlin.edu jcspun@hku.hk

schurch@stanford.edu
pryke@physics.umn.edu

cbischoff@cfa.harvard.edu

ckulesa@email.arizona.edu licai@bao.ac.cn mekarnia@oca.eu

Douglas.Bock@csiro.au info@iloa.org

enzo.pascale@astro.cf.ac.uk Eric.Steinbring@nrc-cnrc.gc.ca Eric.Fossat@oca.eu wg@lamost.org gzhao@nao.cas.cn

gzhao@nao.cas.cn
g.sims@unsw.edu.au glombard@eso.org

g-novak@northwestern.edu durandgs@cea.fr

gong-bo.zhao@port.ac.uk

g.j.murray@durham.ac.uk

g.j.murray@durham.ac.uk
liuguoqing@tsinghuaeducn

francis.halzen@icecube.wisc.edu

hzinnecker@sofia.usra.edu hengz@niaot.ac.cn

h-okita@astr.tohoku.ac.jp

h.matsuo@nao.ac.jp

hcwang@pmo.ac.cn js@cbk.pan.wroc.pl

hamilton@apc.univ-paris7.fr

jean-pierre.rivet@oca.eu

jiyang@pmo.ac.cn

jhwu@bao.ac.cn

jhw ubao.ac.cn sgjin@shao.ac.cn

namjiwoo@gmail.com jcg@mpe.mpg.de

john.dickey@utas.edu.au

j.storey@unsw.edu.au jl@aao.gov.au

w.vernon.jones@nasa.goy

jnavarre@eso.org kara@umd.edu kgoetz@aip.de

jmkovac@cfa.harvard.ed u

guoliang@pmo.ac.cn xyli@niaot.ac.cn lysh@pric.gov.cn

wang@physics.tamu.edu liuly@nao.cas.cn

luc.dame@latmos.ipsl.fr Lyua

inoue@asiaa.sinica.edu.tw rqmao@pmo.ac.cn msarazin@eso.org

smarenssi@dna.gov.ar mwolfire@astro.umd.edu
mjm@esa.int

mark.mcauley@astronomyaustralia.org.au

rmaruyama@wisc.edu seta@physics.px.tsukuba.ac.jp colless@aao.gov.au

m.ashley@unsw.edu.au

m.burton@unsw.edu.au minh@kasi.re.kr

jmould@swin.edu.au imae@nipr.ac.jp

n.tothill@uws.edu.au crouzet@stsci.edu nsharp@nsf.gov 
Halverson Nils

Jay Pasachoff

Rojo Patricio

Koch Patrick

Jones Paul

Chong Pei

Qingyu Peng

Carlberg Raymond

Matsushita Satoki

Lee Seong-Jae

Sheng-Cai Shi

Kazayuki Shiraish

Muchovej Stephen

Ivan Syniavsky

Cheung Sze-leung

Gaisser Thom as

Zhang Tianmeng

Stevenson Toner

Mroczkowski Tony

Pascal Tremblin

Peter Tuthill

Nam Uk-Won

Papitashvili Vladimir

Lingzhi Wang

Jones William

Cui Xiangqun

Jiang Xiaojun

Guan Xin

Zhou Xu

Wu Xuefeng

Chen Xuepeng

Akira Yamaguchi

Gong Yan

Zhang Yanxia

Kang Yong Woo

Omura Yoshiharu

Chu You-Hua

Xiangyan Yuan

Motizuki Yuko

Shang Zhaohui

Zhaowen nils.halverson@ colorado.edu jay.m.pasachoff@william s.ed pato@oan.cl

pmkoch@asiaa.sinica.edu.tw pjones@phys.unsw.edu.au cpei@niaot.ac.cn tpengqy@jnu.edu.cn carlberg@astro.utoronto.ca satoki@asiaa.sinica.edu.tw seong@chungbuk.ac.kr scshi@pmo.ac.cn kshiraishi@nipr.ac.jp sjcm@astro.caltech.edu syn@mao.kiev.ua cheung.szeleung@hku.hk gaisser@bartol.udel.edu zhangtm@nao.cas.cn toners@phm.gov.au tonym@astro.caltech.edu pascal.tremblin@gmail.com p.tuthill@physics.usyd.edu.au uwnam@kasi.re.kr vpapita@nsf.gov lzwang520213@mail.bnu.edu.cn wcjones@princeton.edu xcui@nioat.ac.cn xjjiang@bao.ac.cn guan@ph1.uni-koeln.de zhouxu@bao.ac.cn xfwu@pmo.ac.cn xpchen_yale@hotmail.com yamaguch@nipr.ac.jp ygong1@uci.ed zyx@bao.ac.cn byulmaru@kasi.re.kr omura@rish.kyoto-u.ac.jp yhchu@illinois.edu yyyuan@niaot.ac.cn xyyuan@ niaot.ac.cn
motizuki@riken.jp zshang@gmail.com wzhao7@zjut.edu.cn 


\section{Editorial}

One hundred years ago, on a bright and clear day on December 5, 1912 three young explorers - Francis Bickerton, Alfred Hodgeman and Leslie Whetter - were traversing across the sastrugi in the coastal highlands of Adelie Land in Antarctica, dragging a sledge along behind them. They had just abandoned an 'air tractor' which they were using to pull their load on their journey of discovery, and were struggling across the rough surface. They saw ahead of them a shiny black object, partially buried in the snow. About the size of one's hand, they immediately recognised it to be a meteorite, for how else could it have got there, all alone on top of an immense visage of ice?! They picked it up for their collection and later study. Now known as the Adelie Land Meteorite, and displayed in the Australian Museum in Sydney, it was the first meteorite to be found in Antarctica, though it was to be another 11 years before this find was written up as a scientific paper (Bayly \& Stillwell, 1923). It was the start of astronomy in Antarctica. However, it was to be almost half a century later before another meteorite was found. Indeed, it was not until 1969, when several different types of meteorites were found close together (Nagata, 1975), that it was realised that Antarctica provides a superb platform for finding meteorites on account of the great flows of the ice, gathering objects that fall upon them and taking them to blue-ice fields where the snow is ablated by the wind, leaving them easy to spot and so collect.

Astronomy in Antarctica has had a slow gestation. Cosmic ray studies were the first astronomical science programs, being instigated around the time of the International Geophysical Year (1956-57). It was not until 1979 that the first astronomical science was carrried out at the South Pole, using an optical telescope to measure oscillations in the interior of the Sun (Grec, Fossat \& Pomerantz, 1980). This was followed by a variety of experiments at millimetre-wavelengths looking for interstellar dust and the cosmic microwave background radiation. It was not until the 1990's, with the instigation of CARA, the Center for Astrophysical Research in Antarctica, at the South Pole, that astronomy in Antarctica began to be undertaken in earnest $\dagger$. Since then, the growth in the field has been rapid.

Today astronomical investigations are being carried out at five locations on the Antarctic plateau (South Pole, the ice Domes A, C and F, and at Ridge A), as well as long duration ballooning from McMurdo station on the coast. The images on the following pages provide a flavour of the activity at each of these sites. A rich variety of different types of astrophysics is now being being accomplished using data gathered in Antarctica.

The IAU recognised the potential for astronomy in Antarctica in 1991 at the XXI General Assembly in Buenos Aires, creating the 'Working Group for the Development of Astronomy in Antarctica', under the chair of Peter Gillingham. It was set up as an inter-divisional working group under Division IX (Optical/IR Techniques) and Division $\mathrm{X}$ (Radio Astronomy). Since then meetings on Astronomy in Antarctica have been held at the GA's in The Hague, Kyoto, Sydney, Prague and Rio de Janeiroł. The size and context of these meetings has grown over time, with the last three being designed "Special Sessions", and their proceedings appearing in the IAU's Highlights of Astronomy.

$\dagger$ For a history of astronomy in Antarctica during these early years see Indermuehle, Burton \& Maddison, 2005.

$\ddagger$ See www.phys.unsw.edu.au/jacara/iau/. 
With the XXVIII GA in Beijing in 2012, widespread interest internationally in the prospects for astronomy in Antarctica, and with China beginning the construction of Kunlun Station at the highest point on the Antarctic plateau (Dome A), the time was ripe for the proponents of astronomy in Antarctica to seek to hold an IAU Symposium on this field, and so receive recognition from their peers of the maturity of this field of endeavour. The IAU agreed, and hence was born IAU Symposium 288, "Astrophysics from Antarctica" $\uparrow$. In the pages ahead you will find papers written by the presenters at this Symposium describing a wide variety of activities in a vibrant field.

IAUS288 is the first, but it also may be the last, IAU Symposium devoted to the full subject matter of astronomy in Antarctica. For, having entered the mainstream, the results from Antarctic experiments will be reported more and more at discipline-specific meetings in the future, alongside results from the multitude of facilities that other domains of astronomy bring to the science.

We hope you enjoy reading these Proceedings!

Michael Burton, University of New South Wales

Xiangqun Cui, Nanjing Institute for Astronomical Optics and Technology

Nicholas Tothill, University of Western Sydney

Editors, IAU Symposium 288, "Astrophysics from Antarctica".

\section{References}

Bayly, P. G. W. \& Stillwell, F. L. 1923, The Adelie Land Meteorite, Australasian Antarctic Expedition 1911-14, Scientific Reports Series A, Vol. IV: Geology, Sydney. Publisher A.J. Kent (Sydney)

Grec, G., Fossat, E. \& Pomerantz, M. A. 1980, Nature, 288, 541

Indermuehle, B., Burton, M. G. \& Maddison, S. T. 2005, PASA, 22, 73

Nagata, T., ed. Mem. Nat. Inst. Polar Res., 5 

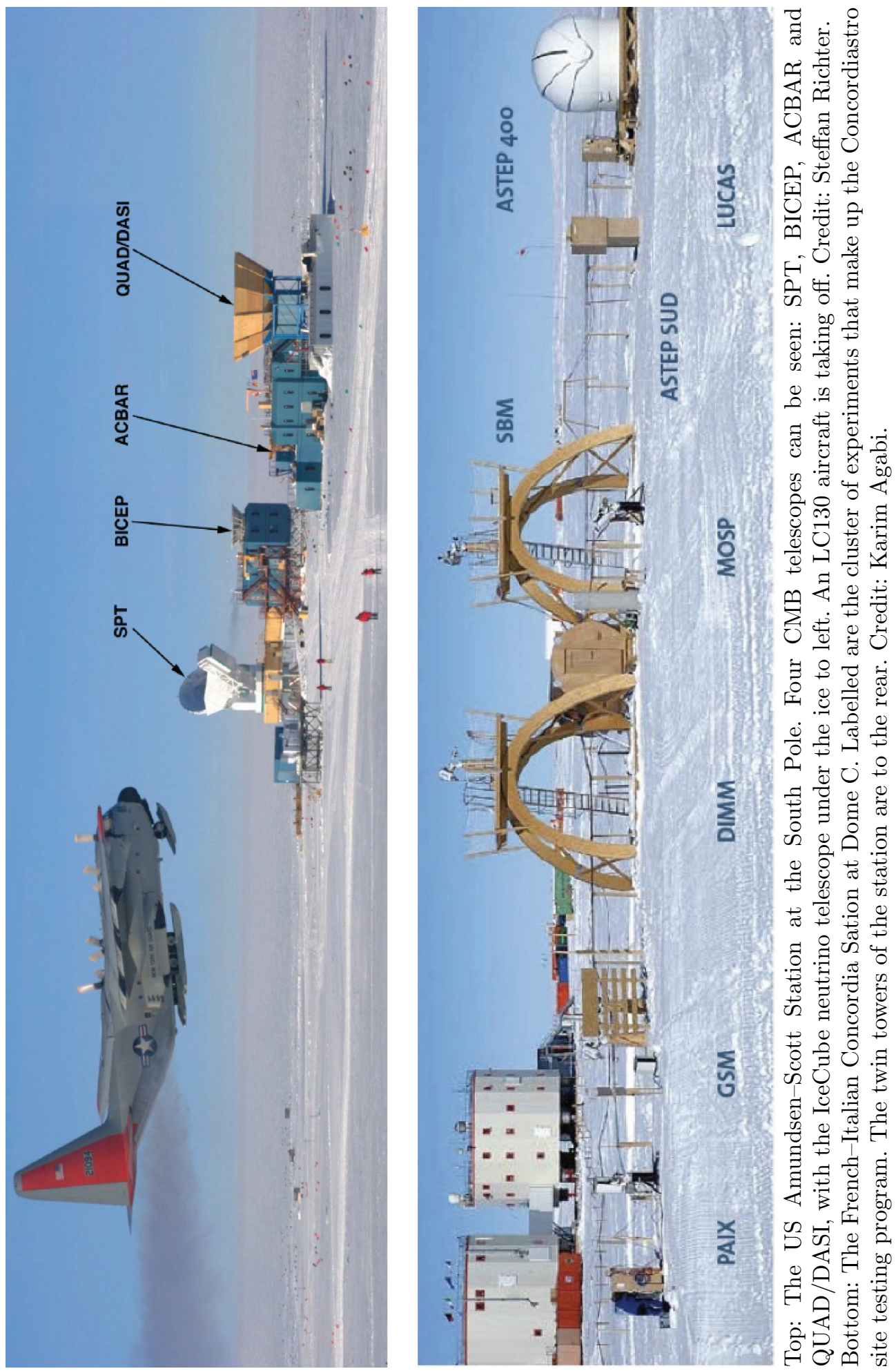


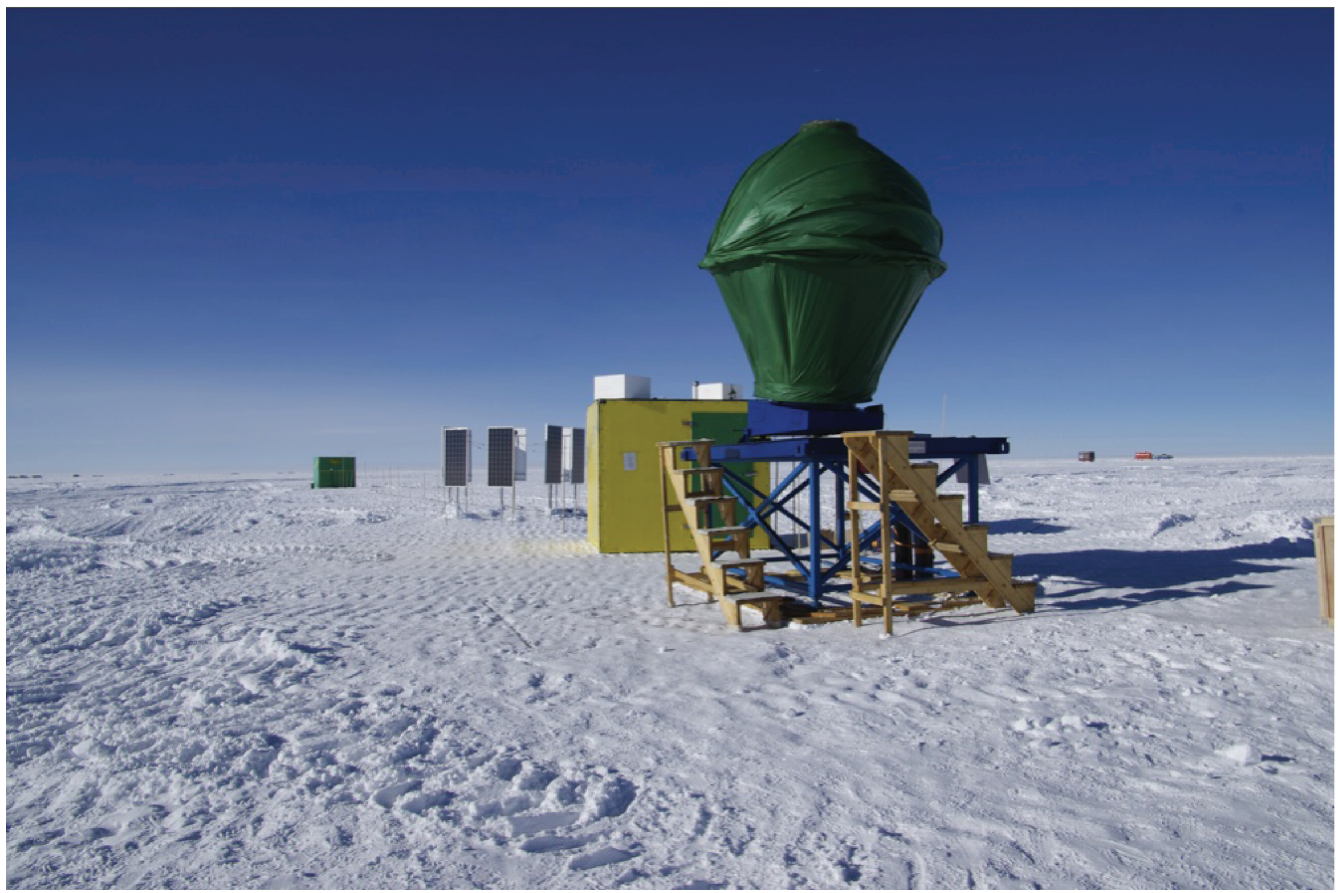

The $0.5 \mathrm{~m}$ AST3 optical telescope at the Chinese Kunlun station at Dome A. The yellow and green buildings behind are the Australian-built PLATO laboratory. Credit: NIAOT.

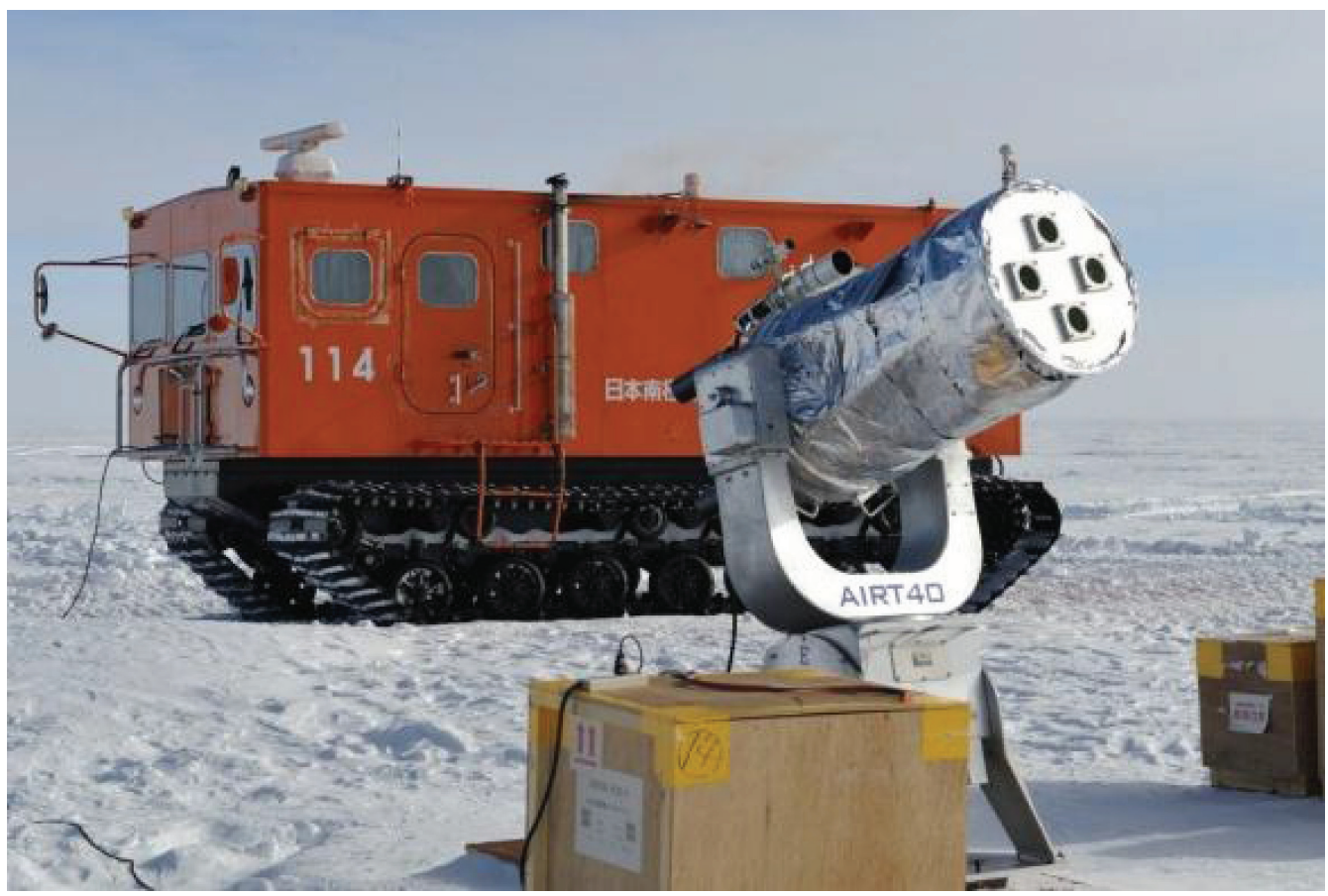

The Tohoku-DIMM placed on the mount for the AIRT $40 \mathrm{~cm}$ infrared telescope at the Japanese Dome Fuji Station at Dome F. Credit: Hirofumi Okita 


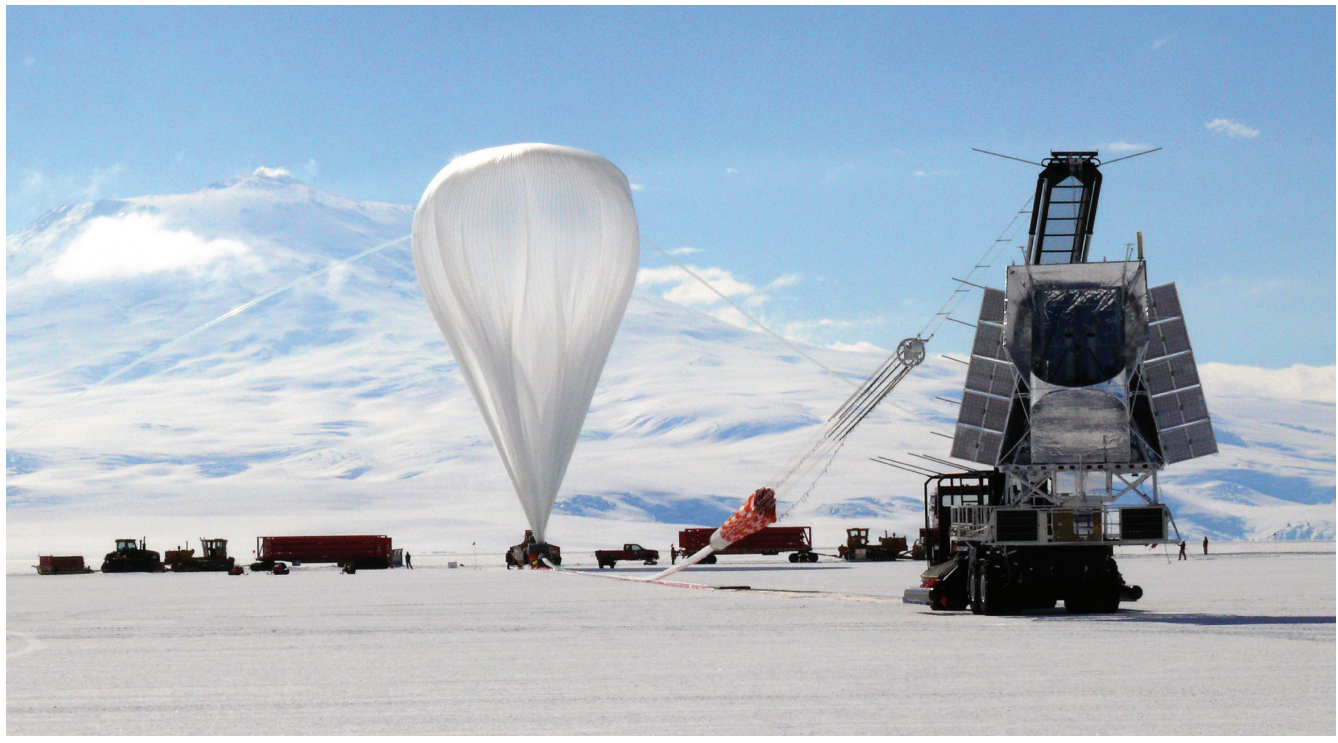

Launch of the $2 \mathrm{~m}$ BLAST THz telescope from the Long Duration Balloon Facility (LDBF) at McMurdo Station. The Mount Erebus volcano is in the background. Credit: Mark Halpern.

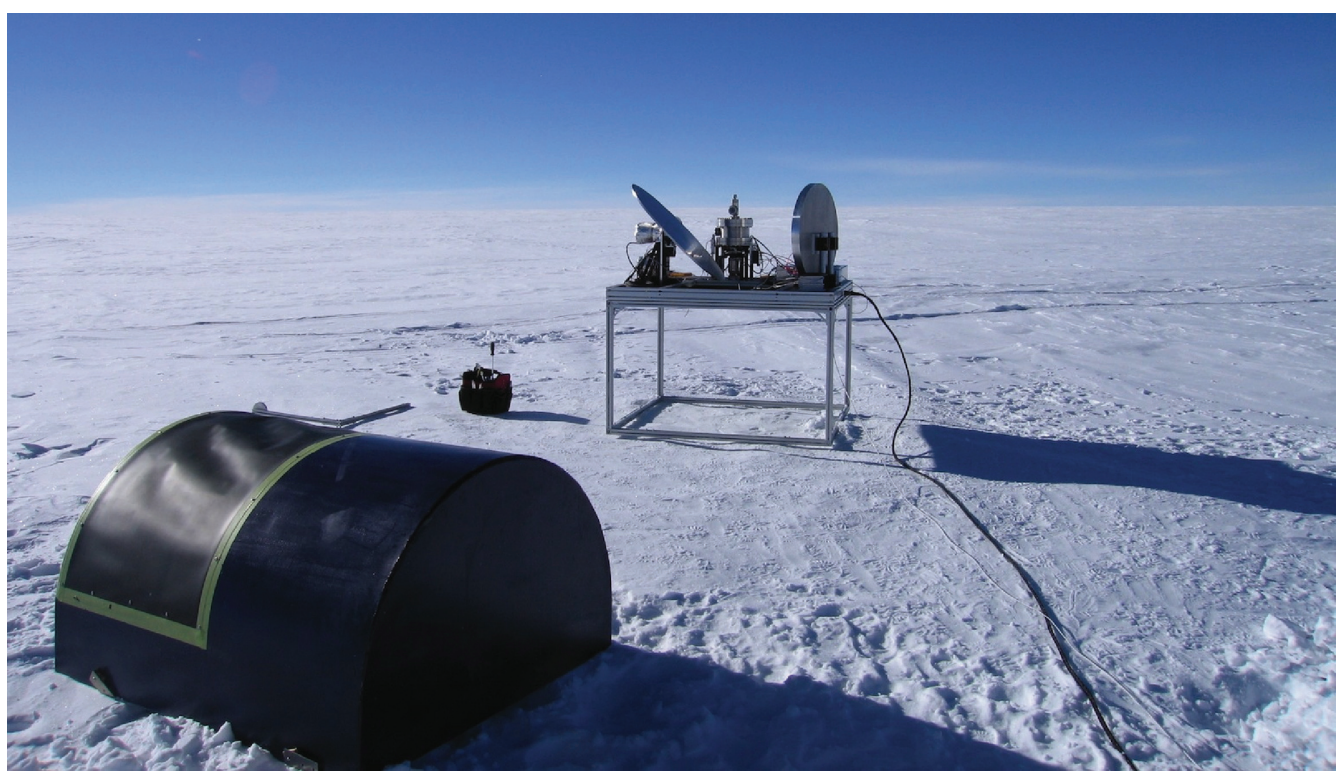

The $60 \mathrm{~cm}$ HEAT THz telescope deployed at the SCAR international station at Ridge A by the USA and Australia. Credit: Craig Kulesa. 